\title{
A SPATIAL-AND-SCALE-DEPENDANT MODEL FOR PREDICTING MSW GENERATION, DIVERSION AND COLLECTION COST BASED ON DWELLING-TYPE DISTRIBUTION
}

\author{
Laurent Spreutels ${ }^{1}$, Martin Héroux ${ }^{2}$ and Robert Legros ${ }^{1, *}$
}

${ }^{1}$ Chaire de Recherche sur la Valorisation des Matières Résiduelles (CRVMR), Department of Chemical Engineering, Polytechnique Montreal, Montreal, Canada

${ }^{2}$ Department of Environment, City of Montreal, Montreal, Canada
Article Info:
Received:
11 July 2019
Revised:
29 April 2020
Accepted:
4 May 2020
Available online:
24 July 2020
Keywords:
Waste generation
Predictive models
Collection cost
Transportation cost
Dwelling-type distribution
Diversion rate

\begin{abstract}
Comprehensive models were developed to predict waste generation for different collection streams. Taking into account the dwelling-type distribution encountered during the different waste collections, it was possible to better capture the waste generation variability. Using the same approach, collection and transportation cost models were also developed. This series of models were validated using data from the Urban Agglomeration of Montreal (UAM), which is composed of 33 districts with widely different scales of population and dwelling characteristics. The unknown parameters of the models were identified through mean square regressions applied on the real data available for the case-study. For example, values of 1.364, 1.019 and $0.500 \mathrm{t} /$ (dwelling.yr) were identified for the total quantity of wastes generated in single-family, duplex and other dwelling, respectively. Using the same approach, it was possible to determine collection time as a function of the dwelling-type distribution along the collection route. Values of $28.7 \mathrm{~s}, 11.4 \mathrm{~s}$ and $5.22 \mathrm{~s}$ were identified as the collection time per dwelling for single-family, duplex and other dwelling, respectively. Equipped with a combination of fitted parameters and reported values from the literature, the models were used as predictive tools. Three features are illustrated in this paper: 1) the simulation of various scales for the generation, diversion and specific collection cost; 2) the effect of adding a new collection stream; 3 ) the effect of an increase of the citizen participation to a specific collection stream. Predicted results enable decision-makers to have access to very useful information.
\end{abstract}

\section{INTRODUCTION}

In the context of municipal solid waste management, it is frequently useful or necessary to estimate the generation and diversion of wastes at different spatial scales (e.g. country, county, city, borough), including at very small scale (e.g. neighbourhood, dwelling, household, individual). Activities where such information becomes essential include the development of future policies (e.g. new collection stream, financial incentive) or public information campaigns adapted to local context and citizen behaviour, as well as waste collection planification or route optimization, in terms of budget, quantities or dwelling-type distribution. All these planning activities are known to be strongly influenced by how MSW are generated and diverted. The case of collection cost is a good example. It is mainly defined by the available or planned collection structure, by the urbanistic characteristics of the collected area (e.g. dwelling-type distribution), by the amount of waste generated in total, and by the diversion of the waste in the collection streams. When the participation of citizens to a particular collection stream increases, this will not only directly influence the collection cost related to this stream, but also the collection cost for other streams.

Usually, data needed to quantify generation and diversion are available only at large scales, and information about collection cost, when publicly available, are difficult to use in different contexts and scales. In the literature, several studies have highlighted the relations between generation, diversion or collection costs and various socio-demographic indicators. Some mathematical models are also described and allow a fair estimation of those parameters. Goel et al. (2017) reviewed the different modeling approaches used in order to forecast solid waste generation rates. For example, some models are integrating geographical information system (GIS) information to predict waste generation rates as a function of household size
* Corresponding author

Robert Legros

email: robert.legros@polymtl.ca 
and other socioeconomic conditions (Purcell and Magette, 2009, Akther et al., 2016). Application of GIS-based methods to link waste generation rate to a multiplicity of factors is increasing as the GIS-linked information becomes more readily available. Although the type of dwellings found in a neighborhood is directly linked to demographic and socioeconomic factors, only one study was found where the waste generation rate was correlated to the dwelling unit size (Grossman et al., 1974). Dwellings types are known to be correlated to the household size, demographic profiles and income level. Since the dwelling-type distribution along a waste collection route can be easily obtained using GIS information, this approach seems to offer a very promising basis for the development of a simple waste prediction tools.

The Chaire de Recherche sur la Valorisation des Matières Résiduelles (CRVMR, Research Chair on Advanced Waste Recovery) at Polytechnique Montreal, is currently developing a methodology to assess the sustainability of Waste Management Systems (WMS), based on the integration of three distinct VMR (for Valorisation des Matières Résiduelles) tools:

VMR-Gen: Agent-Based Model (ABM) to predict the behaviour of the waste generator, providing the MSW flows and compositions of the source-sorted waste streams;

VMR-Sys: Material flow analysis (MFA) based framework to calculate waste and product flows and stocks throughout the WMS. Comprehensive process modules, one for each waste treatment technology, are developed and integrated into this framework;

VMR-Imp: Waste LCA modelling to evaluate the WMS impacts.

To accompany the development of VMR-Gen, we developed a series of predictive models, adaptable to variable scales, to predict the MSW generation, diversion and specific collection costs (in $\$ /$ dwelling and $\$ / t$ ). The main parameters of the model are related to the dwelling-type distribution (e.g. single-family house, duplex, triplex, apartment buildings), which has shown to have a key influence on generation, diversion and collection cost.

In this paper, the models will be described briefly, and key results will be presented and discussed in order to illustrate three features: 1) the simulation of various scales for the generation, diversion and specific collection cost; 2) the effect of adding a new collection stream; 3) the effect of an increase of the citizen participation to a specific collection stream.

\section{MATERIALS AND METHODS}

The following section describes in the first part the waste generation model, which is developed based on the dwelling-type distribution. In the second part, the dwelling-type distribution approach is used to develop a collection and transportation cost model.

The proposed models are developed in order to represent the waste generation, diversion and collection costs on a given territory. The territory used as case-study for this work is that of the Urban Agglomeration of Montreal (UAM), which is constituted by the 19 boroughs of the City of Montreal and 14 linked municipalities. The UAM occupies the entire Island of Montreal, Quebec (Canada).

\subsection{System description}

A territory is described as a spatial zone with the following properties: population, number of dwellings, dwelling-type distribution, distance travelled during one collection, average distances to the waste treatment facilities, annual quantity of waste generated, and annual quantities of waste collected in source-separated streams.

\subsection{Waste stream models}

It is assumed that the generation of waste and the sorting of waste between available source-separated collection streams is constant for a given dwelling-type on a territory. Then, the annual quantity of waste generated on a territory $\left(M_{\text {tot }}\right)$ is expressed as:

$M_{\mathrm{tot}}=\sum_{i \in D} n_{i} m_{\mathrm{tot}}^{i}$

where:

$D$ is the set of dwelling-types (e.g. single-family houses, duplex, other)

$n_{i}$ is the number of dwellings of type $i$ on the territory

$m_{\text {tot }}^{i}$ is the quantity of waste generated annually in a dwelling of type $i$

Let $C$ be the set of source-separated collection streams available on the territory, then, for each $c$ in $C$, the annual quantity of waste collected in the stream $c\left(M_{c}\right)$ is expressed as:

$M_{c}=\sum_{i \in D} n_{i} m_{\mathrm{tot}}^{i} x_{c}^{i} \tau_{c}^{i} \frac{1}{\pi_{c}^{i}} \sigma_{c}^{i}$

where:

$X_{c}^{i}$ is the mass fraction of the waste stream generated that should be placed in collection stream $c$, for a dwelling of type $i$

$\tau_{c}^{i}$ is the recovery rate of collection stream $c$, for a dwelling of type $i$

$\pi_{c}^{i}$ is the purity of collection stream $c$, for a dwelling of type $i$ $\sigma_{c}^{i}$ is the fraction of dwelling of type $i$ having access to collection stream $c$

The residual quantity of waste $\left(M_{\text {RES }}\right)$ is expressed as:

$M_{\mathrm{RES}}=M_{\mathrm{tot}}-\sum_{c \in C} M_{c}$

Finally, the global waste diversion on a territory $(Y)$ is defined as:

$Y=\frac{\sum_{c \in C} M_{C}}{M_{\text {tot }}}$

\subsection{Collection and transportation models}

Based on the modelling approach described by Tanguy, Villot, Glaus, Laforest, \& Hausler (2017), the models for collection and transportation costs are defined in similar ways, considering two main contributions: cost of the fuel and the hourly operating cost for the truck.

For the collection cost model, taking into account the dwelling-type distribution along the route in order to estimate the average collection speed will be considered. It is reasonable to suppose that the required time to pick up 
wastes from one dwelling is a function of the dwelling-type. For example, a single-family dwelling will require a longer collection time than a multiplex dwelling, since in the latter case multiple dwellings are collected during a single stop, hence yielding a shorter collection time per dwelling.

For the transportation costs model, one may assume that these costs are simply related to the distance travelled between the territory where the waste is collected and the final destination. The dwelling-type distribution should not have an effect on these costs.

\subsection{Collection cost}

The annual collection cost for a stream $s(s \in\{C, R E S\}$, meaning source-separated streams or residual stream) is defined as:

$k_{\text {col }}^{s}=\left(k_{\text {fuel,col }}+\frac{k_{h, \mathrm{col}}}{v_{\mathrm{col}}^{S}}\right) d_{\mathrm{col}}^{S} N_{\text {col }}^{S}$

where:

$k_{\text {fuel,col }}$ is the fuel cost per $\mathrm{km}$ for the collection

$k_{h, c o l}$ is the hourly cost of the truck for the collection

$V_{c o l}^{s}$ is the average collection speed for the stream $s$

$d_{c o l}^{s}$ is the distance travelled during one collection of stream $s$

$N_{c o l}^{s}$ is the number of collections of stream $s$ in a year

The average collection speed for the stream $s$ is defined as:

$v_{\mathrm{col}}^{s}=\frac{d_{\mathrm{col}}^{s}}{t_{\mathrm{col}}^{s}}$

where:

$t_{c o l}^{s}$ is the average collection time for the stream s expressed as a function of the dwelling-type distribution:

$t_{\text {col }}^{s}=\sum_{i \in D} n_{i} \alpha_{s}^{i}$

where:

$\alpha_{s}^{i}$ is the average collection time for the stream $s$ in the case of a dwelling of type $i$. For this averaging process, possible covariance terms were not considered. This could be revised in a future improvement of the model.

\subsubsection{Transportation cost}

The annual transportation cost for a stream $s(s \in\{C, R E S\})$ is defined as:

$k_{\text {tran }}^{S}=\left(k_{\text {fuel,tran }}+\frac{k_{h, \text { tran }}}{v_{\text {tran }}^{S}}\right) 2 d_{\text {tran }}^{S} M_{S} \frac{1}{L}$

where:

$k_{\text {fuel,tran }}$ is the fuel cost per $\mathrm{km}$ for the transportation

$k_{h, \text { tran }}$ is the hourly cost of the truck for the transportation

$V_{\text {tran }}^{s}$ is the average transportation speed for the stream $s$

$d_{\text {tran }}^{s}$ is the transportation distance from the centroid of the territory to the destination of stream $s$

$M_{s}$ is the annual quantity of waste collected in the stream $c$

$L$ is the mass capacity of the transportation truck

\subsubsection{Mass and dwelling specific collection and transporta-} tion costs

The mass specific collection $\left(K_{\text {col }}^{s}\right)$ and transportation $\left(K_{\text {tran }}^{\mathrm{s}}\right)$ costs for a stream $s$ are defined as:

$K_{\mathrm{col}}^{S}=\frac{k_{\mathrm{col}}^{S}}{M_{s}}$
$K_{\text {tran }}^{S}=\frac{k_{\text {tran }}^{S}}{M_{S}}$

The dwelling specific collection $\left(\tilde{\mathrm{K}}_{\text {col }}^{\mathrm{s}}\right)$ and transportation $\left(\tilde{\mathrm{K}}_{\text {tran }}^{s}\right)$ costs for a stream $s$ are defined as:

$\widetilde{K}_{\mathrm{col}}^{s}=\frac{k_{\mathrm{col}}^{s}}{\sum_{i \in D} n_{i}}$

$\widetilde{K}_{\text {tran }}^{S}=\frac{k_{\text {tran }}^{s}}{\sum_{i \in D} n_{i}}$

\subsection{Case-study}

Data collected for the Urban Agglomeration of Montreal (UAM) for the year 2016 were used for this case-study. Characteristics of the 33 individual districts (the 19 boroughs of the City of Montreal and 14 linked municipalities) are presented in Table 1. Data presented include the population, the number of dwellings regrouped under three different types: single-family, duplex and other (3 or more apartments), the annual collected weight of total wastes and recyclables. The UAM territory was selected for this case-study because it comprises a wide variety of districts having different scales in terms of population, numbers of dwellings and relative fraction of dwelling-type habitations.

In Figure 1, the number of dwellings and the fraction of single-family houses are presented as a function of the population for the 34 districts of the UAM. For the most populous districts, we observe that the fractions of single-family households are much smaller than for those with lesser population. Trend lines giving the total number of dwellings in a district considering occupancies between 1.5 and 3.0 persons/dwelling are also shown in Figure 1. For districts where there is a high fraction of single-family households, the points are close to the 3.0 occupancy trend line, while the points for districts with high number of other dwellings (multiplex) are close to the 1.5 occupancy trend line. This is consistent with reported data on the average size of household in different types of dwelling in Quebec (2.7 for single-family, 2.2 for duplex and 1.9 for multiplex) (Lagneau, 2018).

\subsection{Model parameters}

Some of the model parameters presented above may be obtained from published waste characterization results. RECYC-QUEBEC publishes a comprehensive report every two years, where it presents the wastes collected in different streams, including household wastes, recyclable materials (REC) and organic wastes (ORG) collected in the Province of Quebec. Since the UAM data are part of those results, the reported values from RECYC-QUEBEC were used to estimate some parameters in our generation models. These parameters and the reported values are shown in Table 2.

The fuel costs per km and the hourly costs are considered constant for both collection and transportation and equal to $0.89 \$ / \mathrm{km}$ and $72 \$ / \mathrm{km}$, respectively. The mass capacity of the transportation truck is fixed to $10 \mathrm{t}$.

\section{RESULTS AND DISCUSSION}

The unknown parameters of the model $\left(m_{\mathrm{tot}}^{i}\right.$ and $\left.\alpha_{\mathrm{RES}}^{\mathrm{i}}\right)$ were identified through mean square regressions applied on the real data available for the case study. The fitted pa- 
TABLE 1: Characteristics of the 33 districts of the UAM in 2016 (Montréal, 2017).

\begin{tabular}{|c|c|c|c|c|c|c|}
\hline District & Population & $\mathbf{n}^{\text {single }}$ & $\mathrm{n}^{\text {duplex }}$ & $n^{\text {other }}$ & $M_{\text {tot }}[t / y r]$ & $M_{R E C}[t / y r]$ \\
\hline $\mathrm{AC}$ & 136461 & 8372 & 15781 & 38346 & 46479 & 8762 \\
\hline AJ & 44567 & 3152 & 6039 & 10288 & 14775 & 2666 \\
\hline $\mathrm{BF}$ & 19801 & 6264 & 8 & 642 & 9870 & 2268 \\
\hline $\mathrm{BU}$ & 3900 & 1324 & 2 & 46 & 2096 & 401 \\
\hline BV & 4980 & 989 & 293 & 850 & 2251 & 558 \\
\hline $\mathrm{CL}$ & 33847 & 4113 & 1334 & 9044 & 11819 & 2169 \\
\hline $\mathrm{CN}$ & 172961 & 6483 & 19160 & 57941 & 56038 & 11698 \\
\hline DO & 50789 & 12461 & 202 & 4919 & 20809 & 3717 \\
\hline DV & 19431 & 4334 & 372 & 4291 & 7822 & 1912 \\
\hline $\mathrm{HS}$ & 7279 & 1480 & 529 & 658 & 2641 & 639 \\
\hline IS & 19123 & 4885 & 471 & 1536 & 8576 & 1766 \\
\hline $\mathrm{KL}$ & 21270 & 6325 & 14 & 463 & 9230 & 2184 \\
\hline LC & 45003 & 4802 & 4370 & 12610 & 17598 & 3403 \\
\hline LN & 81777 & 2933 & 9065 & 19947 & 27990 & 5183 \\
\hline LR & 101530 & 9412 & 5843 & 24921 & 31539 & 6479 \\
\hline LS & 79651 & 3724 & 16681 & 16322 & 26365 & 6309 \\
\hline ME & 3846 & 510 & 408 & 924 & 1916 & 300 \\
\hline $\mathrm{MH}$ & 139612 & 6477 & 16840 & 47944 & 49656 & 10844 \\
\hline $\mathrm{MN}$ & 89145 & 4547 & 10759 & 21318 & 30048 & 6000 \\
\hline MO & 5212 & 1218 & 579 & 174 & 2192 & 454 \\
\hline MR & 20869 & 4156 & 739 & 3043 & 10323 & 2126 \\
\hline $\mathrm{OM}$ & 25043 & 1985 & 1144 & 6694 & 8920 & 2182 \\
\hline PC & 31898 & 8572 & 229 & 3756 & 13770 & 3617 \\
\hline PM & 105139 & 1865 & 5973 & 57432 & 38712 & 8967 \\
\hline PR & 72399 & 16073 & 1185 & 9044 & 26974 & 5499 \\
\hline RO & 142578 & 3578 & 14404 & 60138 & 49614 & 12523 \\
\hline $\mathrm{RP}$ & 111617 & 21556 & 4663 & 16743 & 40832 & 8717 \\
\hline so & 78027 & 2804 & 7528 & 32595 & 28179 & 6356 \\
\hline SV & 929 & 346 & 10 & 7 & 577 & 127 \\
\hline VD & 70527 & 2933 & 4169 & 29457 & 24122 & 6626 \\
\hline VM & 88799 & 1548 & 1762 & 59209 & 30719 & 8633 \\
\hline VS & 149075 & 3819 & 18858 & 47162 & 49562 & 7931 \\
\hline WM & 20621 & 3563 & 579 & 4794 & 8968 & 1917 \\
\hline
\end{tabular}

rameters together with the parameters presented in Table 2 are then used to solve the model in order to simulate the waste generation and collection for each district of the case-study. The results of the simulations are presented and discussed in this section.

\subsection{Generated stream}

The values of $1.364,1.019$ and $0.500 \mathrm{t} /$ (dwelling.yr) have been identified for $m_{\text {tot }}^{\text {single }}, m_{\text {tot }}^{\text {duplex }}$ and $m_{\text {tot }}^{\text {other }}$, respectively. The comparison of the predicted generated quantities with the real quantities is presented in Figure 2. A very good agreement between predicted and real values is observed. Figure 2 shows how the predictions of MSW generation are improved when the distribution of dwelling-types along the collection route is taken into account $\left(R^{2}=99 \%\right)$, compared to predictions using a model based solely on the total number of dwellings being collected and a unique average value for $m_{\text {tot }}$ equal to $0.698 \mathrm{t} /$ (dwelling.yr) $\left(R^{2}=92 \%\right)$. This figure clearly shows that the dwelling-type distribution has a real influence on the quantities of MSW being generated along a collection route, as quantified by the values of $m_{\text {tot }}^{i}$ and that averaging the generation per collected dwelling does not capture this difference.

\subsection{Collected stream}

In the case of the source-separated collection stream of recyclables (REC), the comparison between predicted and real quantities of collected recyclables is presented in Figure 3. It is worth mentioning that in this case, we only used values reported in the literature for the model param- 


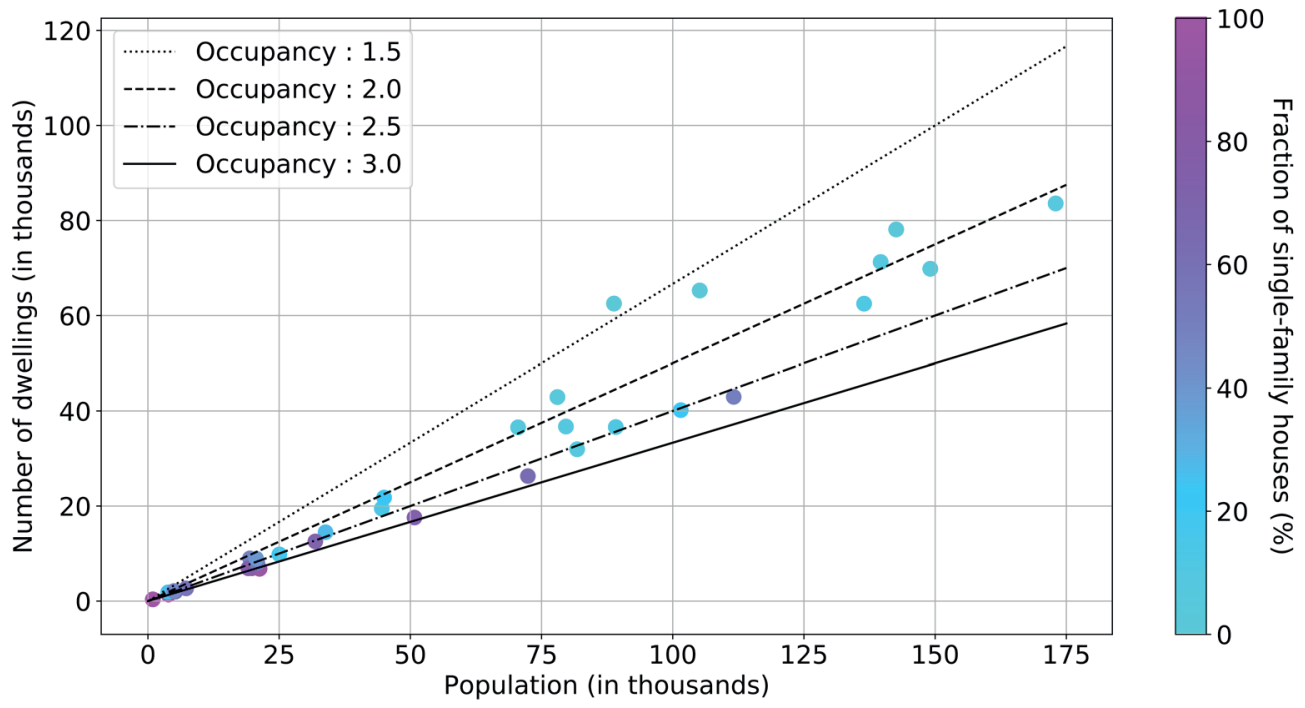

FIGURE 1: Number of dwellings and fraction of single-family dwellings as a function of the population for the 33 districts of the UAM (trend lines show the number of dwellings as a function of the population in the districts, for different occupancies).

eters. Again, a very good agreement between the predicted and the real values is observed, although a greater dispersion in the resulting fit is seen.

Since the deployment of the source-separated collection stream for the organics (ORG) is still recent and in progress, it is not possible to compare the model predictions for this stream with real data as they are not available for all districts. Consequently, this stream is combined with the residual stream. The comparison of the predicted collected quantities with the real quantities, in the case of the combination of the residual stream with the source-separated organics collection, is presented in Figure 4.

The fitted parameters for each collection type and dwelling type are in reasonable agreement with published results from RECYC-QUEBEC. From the generation model, it is possible to estimate the fraction of total waste that is being diverted in the other source-separated collection streams (recyclables and organic wastes where available). A global diversion rate of $22 \%$ on average is observed for all UAM districts. Results presented so far confirm that using an approach that takes into account the types of dwellings encountered along the collection route provides a more accurate predictive model of waste generation and diversion.

\subsection{Collection and transportation costs}

Since the objective here is to estimate the collection and transportation costs without including profit margin

TABLE 2: Model parameters obtained from reported values in the literature (Lagneau, 2018).

\begin{tabular}{c|c|c|c} 
Parameter & Single-family & Duplex & Other \\
\hline$X_{R E C}^{i}$ & $32.3 \%$ & $33.1 \%$ & $36.8 \%$ \\
\hline$X_{O R G}^{i}$ & $43.3 \%$ & $32.3 \%$ & $33.6 \%$ \\
\hline$\tau_{R E C}^{i}$ & $65 \%$ & $60 \%$ & $48 \%$ \\
\hline$\pi_{R E C}^{i}$ & $90 \%$ & $90 \%$ & $85 \%$ \\
\hline$\sigma_{R E C}^{i}$ & $100 \%$ & $100 \%$ & $100 \%$ \\
\hline
\end{tabular}

and other fixed costs that would be charged by a private waste collector, it is not possible to compare these cost estimates with the actual contractual costs. Only the variations from one district to another and the influence of the source-sorting behaviour of the citizens on the trends and orders of magnitudes of these predicted costs will be discussed.

As demonstrated above, the use of the dwelling-type distribution as the basis of our generation predictive model gave good results. The same approach was used to determine collection time as a function of the dwelling-type distribution along the collection route. Using data from a number of collection sectors where the residual waste collection trucks were equipped with GPS (see Table 3), we were able to identify the values of $0.00797 \mathrm{~h}(28.7 \mathrm{~s})$,

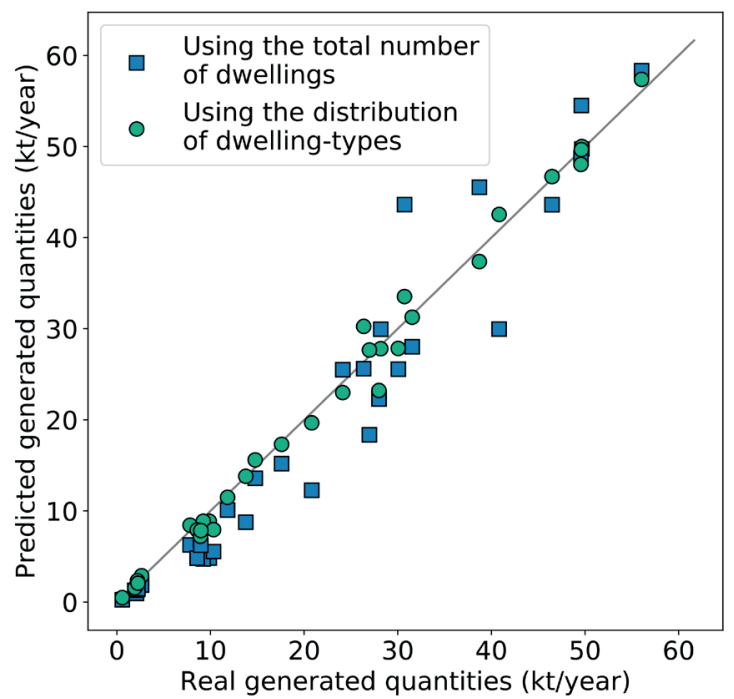

FIGURE 2: Comparison between predicted and real quantities of total generated wastes (Mtot) for the UAM districts based on two approaches: (green circles) using the dwelling-type distribution; (blue squares) using the total number of dwellings. 


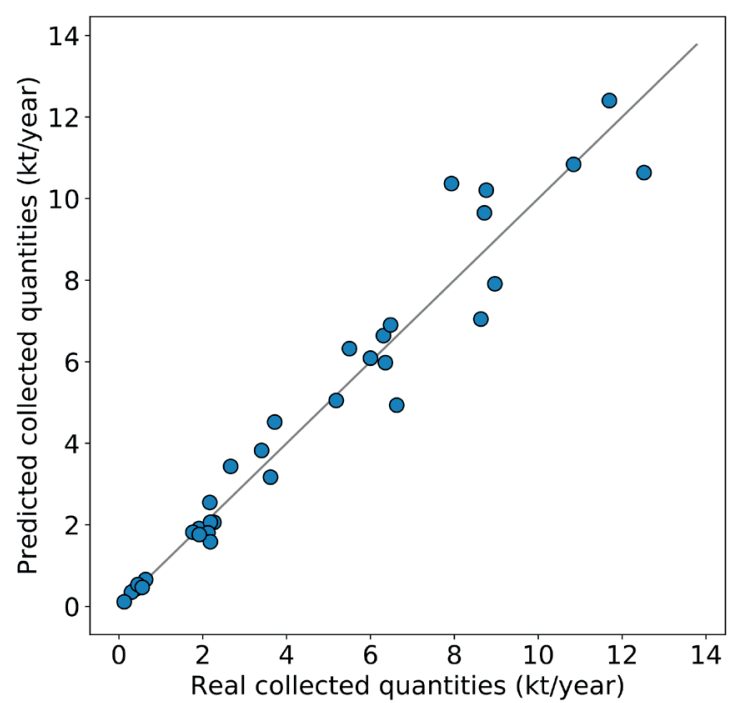

FIGURE 3: Comparison between predicted and real quantities of collected recyclables (MREC) for the UAM districts, based on the dwelling-type distribution.

$0.00317 \mathrm{~h}(11.4 \mathrm{~s})$ and $0.00145 \mathrm{~h}(5.22 \mathrm{~s})$ for $\alpha_{R E S}^{\text {single }}, \alpha_{R E S}^{\text {duplex- }}$ and $\alpha_{R E S}^{\text {other }}$, respectively.

The comparison between predicted and real collection times is presented in Figure 5. In this Figure, fitted results using the total number of dwellings, with a unique average collection time per dwelling equal to $0.00272 \mathrm{~h}$ (9.8 s), are also presented. It is clear once again that the predictions using a model based on the dwelling-type distribution are more accurate $\left(R^{2}=94.6 \%\right)$ than when only the number of dwellings is considered $\left(R^{2}=-14.6 \%\right)$. In this case, the influence is captured through the collection time, which is specific to each type of dwelling configuration. The parameters used in the models were adjusted for the Province of Quebec, Canada, but they could easily be adapted to other regional contexts.

The values of $\alpha_{R E S}^{i}$ were then used to estimate residual waste collection times for 18 selected districts of the UAM for which data were available. Knowing the total annual

TABLE 3: Dwelling-type distribution and residual waste (RES) collection times for 12 collection sectors of the UAM.

\begin{tabular}{c|c|c|c|c} 
Collection sector & $\mathbf{n}^{\text {single }}$ & $\mathbf{n}^{\text {duplex }}$ & $\mathbf{n}^{\text {other }}$ & [h] \\
\hline LC-1 & 2835 & 1015 & 4295 & 32.6 \\
\hline LS-1 & 1215 & 4060 & 1925 & 25.0 \\
\hline LS-2 & 770 & 4985 & 1380 & 21.6 \\
\hline LS-3 & 865 & 3205 & 4230 & 24.1 \\
\hline LS-4 & 1100 & 5250 & 3070 & 31.3 \\
\hline MN-1 & 1115 & 1925 & 5640 & 25.2 \\
\hline MN-2 & 1895 & 1395 & 6135 & 27.0 \\
\hline MN-3 & 420 & 405 & 7150 & 15.5 \\
\hline LN-1 & 1970 & 690 & 9540 & 31.0 \\
\hline LN-2 & 535 & 2195 & 10850 & 26.1 \\
\hline SO-1 & 375 & 2245 & 5145 & 18.1 \\
\hline SO-2 & 690 & 3335 & 2350 & 20.0 \\
\hline
\end{tabular}

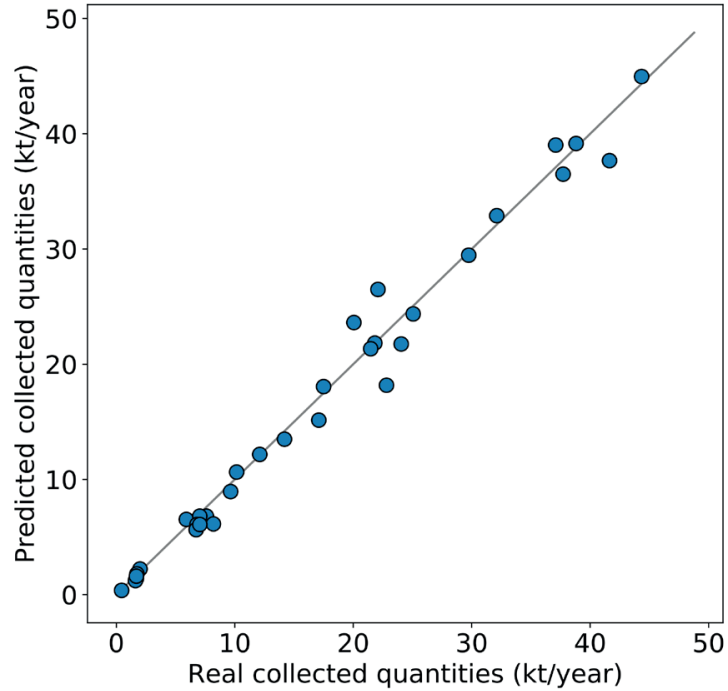

FIGURE 4: Comparison between predicted and real quantities of residual wastes (MRES + MORG) for the UAM districts, based on the dwelling-type distribution.

collection distances and the annual numbers of residual waste collections for each district (see Table 4), it was possible, using the model developed in section 2.3 , to calculate the average collection velocity and the collection cost for each of them. In a similar manner, knowing the transportation distance between each of the selected districts and the final destination, together with the average transportation velocity (see Table 4), it was possible to estimate the transportation cost. These costs are presented in Figure 6 and are reported on two specific bases: $\$ / d$ welling and $\$ /$ t.

Since the districts have a wide range of population and distances being covered for collection and transportation of the residual wastes, the specific costs vary greatly from one district to another. The collection costs vary between 8 \$dwelling and $24 \$ /$ dwelling (12 \$/t to $44 \$ / t)$ and transportation costs vary between $2 \$ /$ dwelling and 11 $\$ /$ dwelling (4 $\$ / t$ to $16 \$ / t)$. These wide ranges of values confirm the importance of taking into account not only the distances for collection and transportation, but also the distribution of dwelling-types encountered during the collection. Overall, transportation costs are much lower than collection costs with a ratio of about 3 between the two in terms of $\$ / t$.

\subsection{Addition of a new collection and variation of the participation}

So far, predictive models to estimate generated quantities of wastes for different collection streams and to estimate collection and transportation costs were presented. The model parameters were determined through a combination of fitted variables and reported values in the literature. Predictions from these models were compared to actual data from 33 districts having very different profiles (see Table 1) and results are very promising.

In this section, the models will be used as prospective tools to determine the impact of adding a new collection stream, a source-separated organic waste collection 


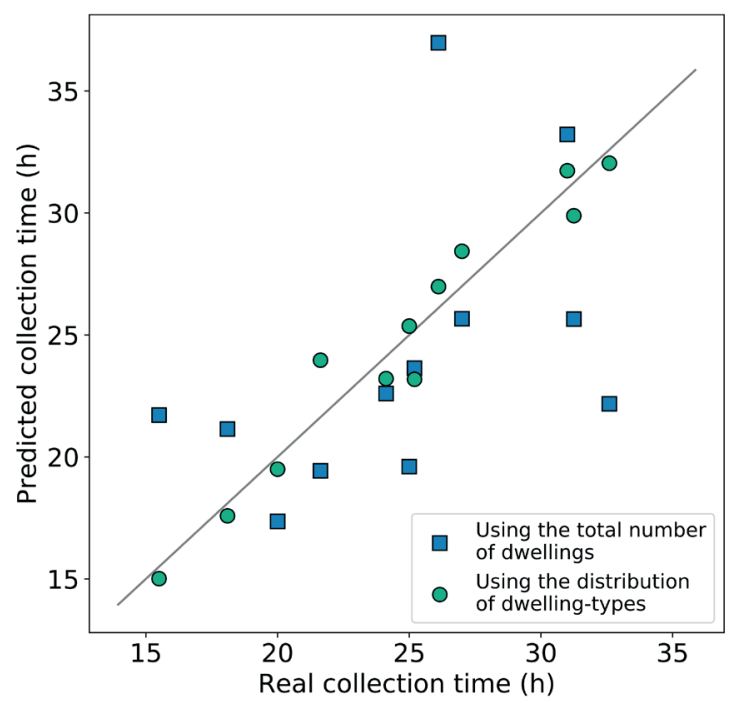

FIGURE 5: Comparison between predicted and real residual waste (RES) collection time for the UAM districts based on two approaches: (green circles) using the dwelling-type distribution; (blue squares) using the total number of dwellings.

(ORG), on the global diversion rate and collection cost. In order to simulate this new addition, the recovery rate $\tau_{\mathrm{ORG}}^{\mathrm{i}}$ was varied, as well as the percentage of dwelling having access to this new collection.

The simulation is based on the following assumptions:

- A source-separated organic waste collection stream is added in the 33 districts;

- The mass fraction of the total waste stream generated that should be placed in the organic waste collection

TABLE 4: Characteristics of the 18 selected districts regarding the collection and transportation of the residual stream.

\begin{tabular}{|c|c|c|c|c|}
\hline Territory & $\mathrm{d}_{\text {col }}^{\mathrm{RES}}[\mathrm{km}]$ & $\mathbf{N}_{\text {col }}^{\text {RES }}[-]$ & $\mathrm{d}_{\text {tran }}^{\mathrm{RES}}[\mathrm{km}]$ & $\mathbf{v}_{\text {tran }}^{\mathrm{RES}}[\mathbf{k m} / \mathbf{h}]$ \\
\hline$A C$ & 696 & 104 & 14 & 51 \\
\hline AJ & 304 & 104 & 19 & 68 \\
\hline $\mathrm{CN}$ & 560 & 104 & 23 & 52 \\
\hline IS & 237 & 52 & 26 & 51 \\
\hline LC & 298 & 52 & 25 & 62 \\
\hline LN & 386 & 78 & 9 & 60 \\
\hline LR & 773 & 52 & 17 & 61 \\
\hline LS & 403 & 104 & 27 & 44 \\
\hline $\mathrm{MH}$ & 340 & 52 & 12 & 71 \\
\hline MN & 372 & 104 & 24 & 64 \\
\hline MO & 95 & 104 & 34 & 57 \\
\hline PM & 309 & 104 & 18 & 46 \\
\hline PR & 565 & 52 & 22 & 56 \\
\hline $\mathrm{RP}$ & 484 & 52 & 15 & 65 \\
\hline so & 259 & 52 & 29 & 53 \\
\hline VD & 239 & 52 & 30 & 51 \\
\hline VM & 397 & 104 & 33 & 51 \\
\hline VS & 509 & 104 & 12 & 60 \\
\hline
\end{tabular}

stream $\left(x_{O R G}^{i}\right.$ is taken as reported values from the literature (see Table 2);

- The purity of this organic waste collected stream $\left(\pi_{O R G}^{i}\right)$ is $100 \%$;

- The recovery rate $\left(\tau_{O R G}^{i}\right)$, equivalent to the participation rate of the citizens to the new collection, is varied from 0 to $100 \%$;

- The fraction of dwellings having access to this new collection stream ( $\sigma_{O R G}^{i}$ is modulated according to two scenarios: 1. all dwellings have access to the new collection ( $\sigma_{O R G}^{i}=100 \%$ for all the dwellings); 2 . Only single-family and duplex dwellings have access to the new collection and not the others $\left(\sigma_{O R G}^{i}=100 \%\right.$ for single-family and duplex, $\sigma_{O R G}^{i}=0 \%$ for other).

Results are presented in Figure 7 in terms of the global diversion rate ( $\mathrm{Y}$, see equation 4$)$ as a function of the participation rate for the two scenarios.

In Figure 7, the average value for each scenario is shown as a trend line, while the variability associated with the different districts is shown as a colored area. When all dwellings have access to the new organic waste collection, the global diversion rate is directly related to the participation rate with little variability amongst the different districts (blue area in Figure 7 is quite narrow). This is not the case when only single-family houses and duplexes have access to the new collection, in which case there is much wider differences in diversion rate between the different districts (wider orange area).

The cost models presented above were used to estimate the specific collection costs for the residual waste (RES) collection resulting from the addition of the new organic stream collection. The results are presented in Figure 8 , where the specific costs in $\$ / d$ welling and $\$ / t$ for the residual waste collection stream are plotted as a function of participation rate for the same two scenarios.

Figure 8 shows the impact of implementing a new collection stream (ORG) on the collection and transportation costs of an existing collection (RES). In this case, the specific costs for the residual collection, in \$/dwelling and in $\$ / t$, are presented as a function of participation rate in the new organic waste collection. As expected, the collection costs of residual waste in $\$ /$ dwelling is not affected by the presence of the new collection for both scenarios, since the trucks collecting the waste must travel the same distance as before (blue and orange lines on left graph). However, when the collection costs are expressed in $\$ / t$, since some of the waste is now diverted into the new organic collection, there is a strong dependency on the participation rate (blue and orange curves on right graph). For the transportation costs, they go down slightly in all cases since there are less waste to transport. It is interesting to note that the trend is reverse between the two scenarios, with the transportation cost in \$/dwelling being more affected by the participation rate when all dwellings have access to the new collection, while the transportation cost in $\$ / t$ is more affected by the participation rate in the case where only single-family houses and duplexes have access to the new collection. This type of behaviour is certainly very valuable information to take 


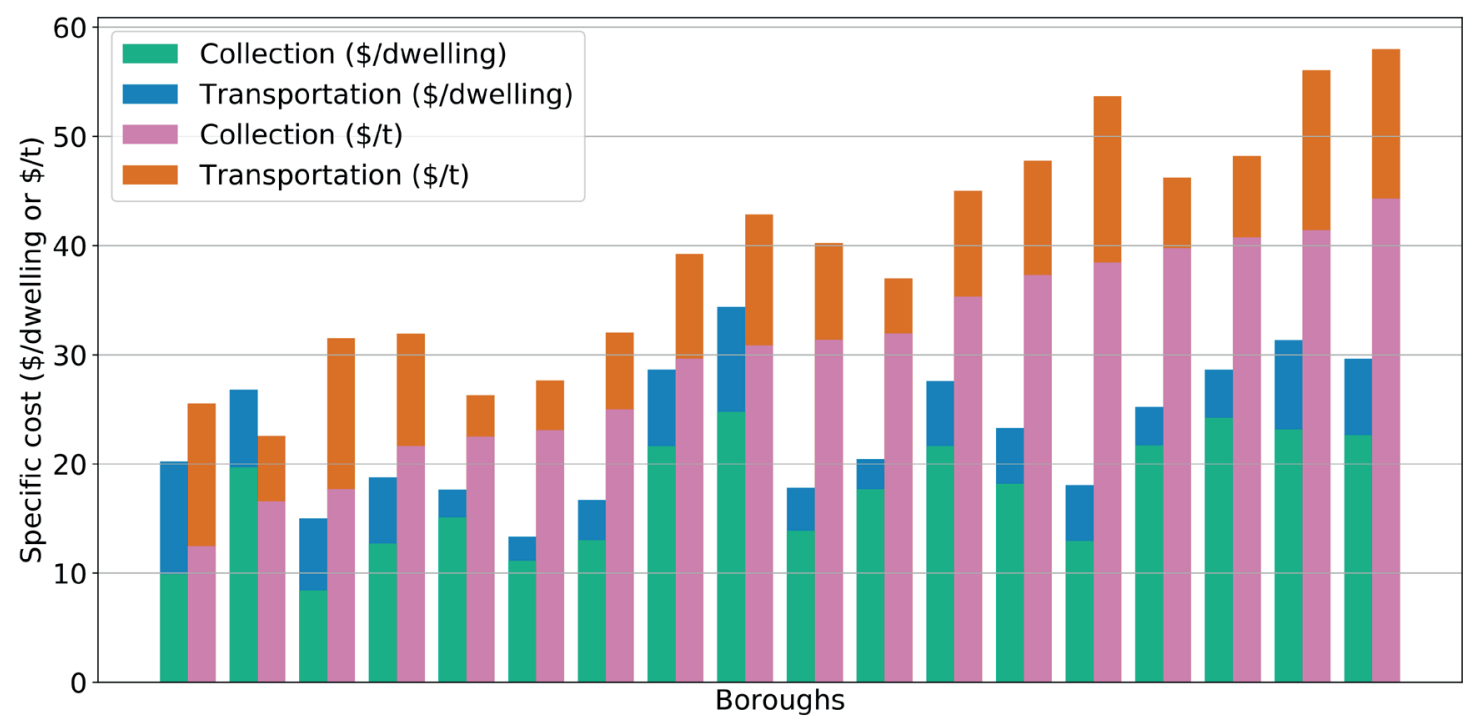

FIGURE 6: Specific collection and transportation costs per dwelling and per tonne as predicted by the model for the 18 selected districts of UAM.

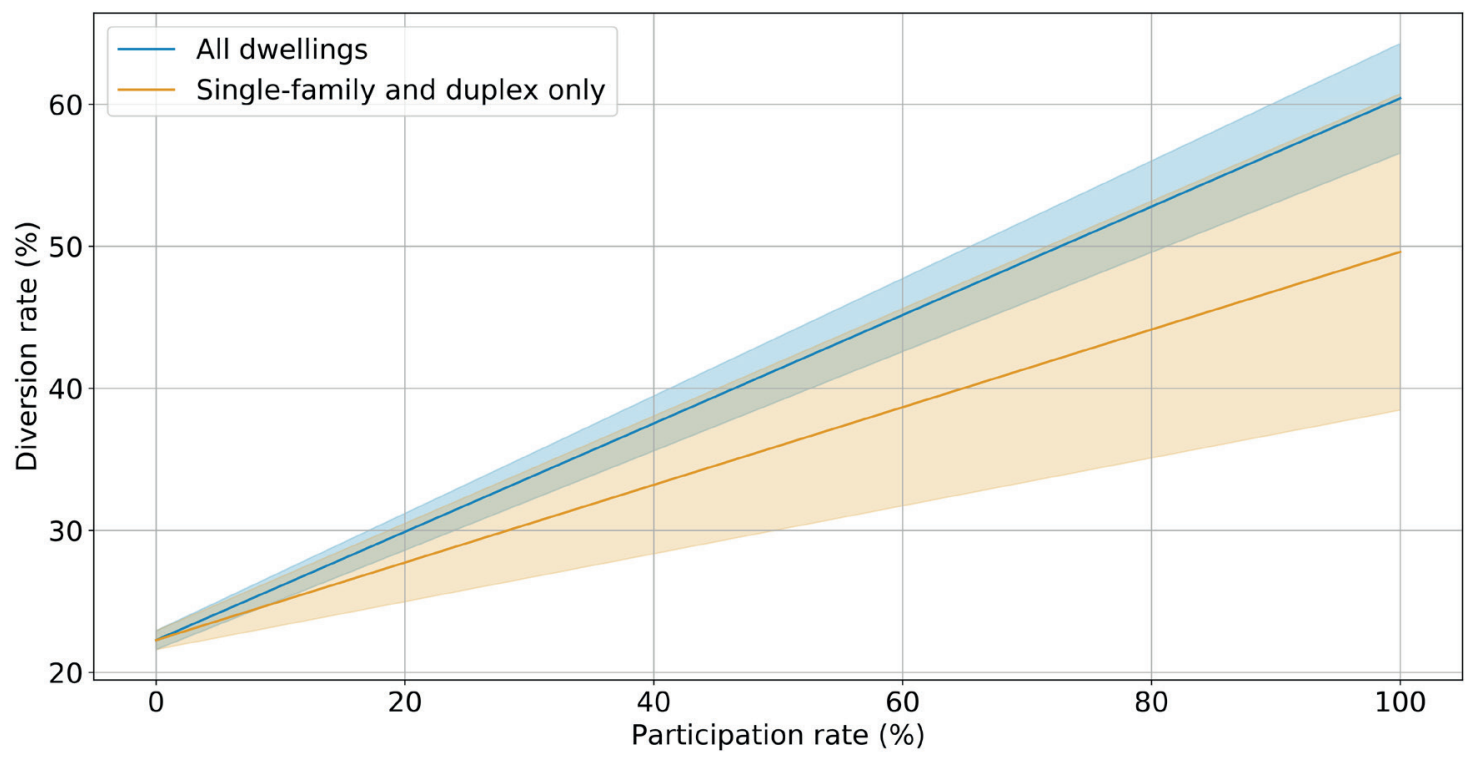

FIGURE 7: Global diversion rate as a function of the participation rate in a new collection stream of organic wastes (in blue: all the dwellings have access; in orange: only the single-family and the duplex dwellings have access).

into account when new collection streams are being evaluated.

\section{CONCLUSIONS}

As part of the development of decision-making tools by the CRVMR, a series of comprehensive models were developed to estimate waste generation and diversion, as well as collection/transportation costs. These models make use of the dwelling-type distribution encountered during the collection of different source-separated waste streams. They were calibrated and validated for widely different profiles of districts in terms of scales of population and dwelling characteristics. Using this approach, we were able to capture the effect of the dwelling-type distribution on the generation and diversion of wastes. This led to better predictive models and in turn, to better estimates of collection and transportation costs. The models were then used to simulate the effect of adding a new organic waste collection, with different participation rate of the population to this new collection. Prediction results revealed complex interactions. The model results give decision-makers very useful information in several of their tasks, such as allocation of collection contracts, estimation of scale for waste treatment facility and implementation of waste-related incentives.

\section{AKNOWLED GEMENTS}

The authors would like to thank Jérémy Lagneau for his contribution to the development of the methodology reported in this paper. Also, the financial support from the 

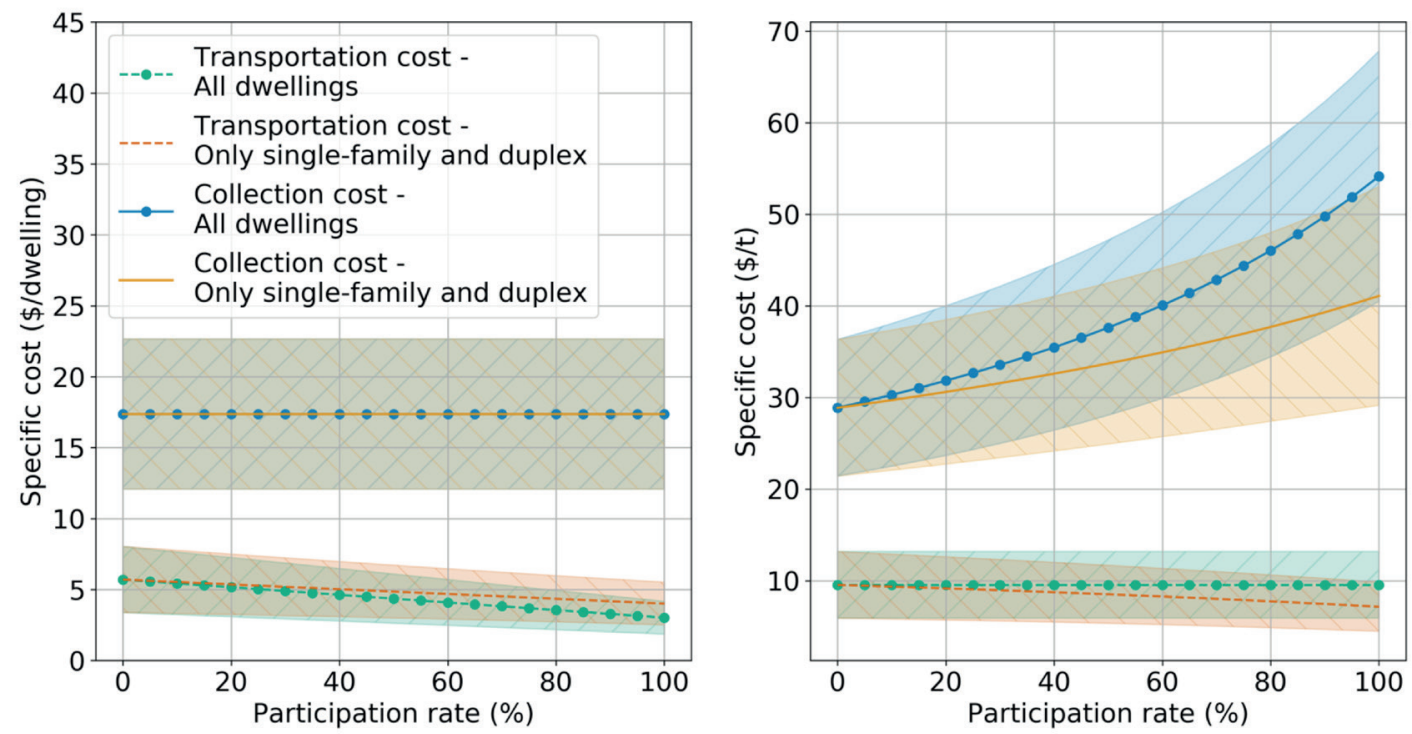

FIGURE 8: Specific costs of the residual waste (RES) collection as a function of the participation rate in a new collection of organic wastes (in blue: all the dwellings have access; in orange: only the single-family and the duplex dwellings have access).

CRVMR partners is greatly appreciated: City of Montreal, City of Laval, City of Gatineau and RECYC-QUEBEC.

\section{REFERENCES}

Akther, A., Ahamed, T., Takigawa, T., Noguchi, R (2016). GIS-based multi-criteria analysis for urban waste management. Journal of the Japan Institute of Energy 95(5):457-467

Goel S., Ranjan V.P., Bardhan B., Hazra T. (2017). Forecasting Solid Waste Generation Rates. In: Sengupta D., Agrahari S. (eds) Modelling Trends in Solid and Hazardous Waste Management. Springer, Singapore

Grossman D, Hudson JF, Marks DH (1974). Waste generation models for solid waste collection. Journal of the Environmental Engineering Division, 100, 1219-1230
Lagneau, J. (2018). Étude multi-échelles des coûts de gestion de la matière résiduelle organique au Québec (Masters thesis, École Polytechnique de Montréal). Retrieved from https://publications. polymtl.ca/3673/

Montréal (2017). Portrait 2016 des matières résiduelles de l'agglomération de Montréal. Retrieved from http://collections.banq.qc.ca/ ark:/52327/2239873

Purcell M, Magette WL (2009). Prediction of household and commercial BMW generation according to socio-economic and other factors for the Dublin region. Waste Management, 29, 1237-1250.

Tanguy, A., Villot, J., Glaus, M., Laforest, V., \& Hausler, R. (2017). Service area size assessment for evaluating the spatial scale of solid waste recovery chains: A territorial perspective. Waste Management, 64, 386-396. https://doi.org/10.1016/j.wasman.2017.03.027 